\title{
Disorder, critical currents, and vortex pinning energies in isovalently substituted $\mathrm{BaFe}_{2}\left(\mathrm{As}_{1-x} \mathrm{P}_{x}\right)_{2}$
}

\author{
S. Demirdiş \\ Laboratoire des Solides Irradiés, CNRS UMR 7642 \& CEA-DSM-IRAMIS, Ecole Polytechnique, F-91128 Palaiseau cedex, France \\ Y. Fasano \\ Laboratorio de Bajas Temperaturas, Centro Atómico Bariloche \& Instituto Balseiro, Avenida Bustillo 9500, 8400 Bariloche, Argentina
}

S. Kasahara and T. Terashima

Research Center for Low Temperature \& Materials Sciences, Kyoto University, Sakyo-ku, Kyoto 606-8501, Japan

T. Shibauchi and Y. Matsuda

Department of Physics, Kyoto University, Sakyo-ku, Kyoto 606-8502, Japan

Marcin Konczykowski

Laboratoire des Solides Irradiés, CNRS UMR 7642 \& CEA-DSM-IRAMIS, Ecole Polytechnique, F-91128 Palaiseau cedex, France

\author{
H. Pastoriza \\ Laboratorio de Bajas Temperaturas, Centro Atómico Bariloche \& Instituto Balseiro, Avenida Bustillo 9500, 8400 Bariloche, \\ Rio Negro, Argentina \\ C. J. van der Beek \\ Laboratoire des Solides Irradiés, CNRS UMR 7642 \& CEA-DSM-IRAMIS, Ecole Polytechnique, F 91128 Palaiseau cedex, France
}

(Received 10 January 2013; published 11 March 2013)

\begin{abstract}
We present a comprehensive overview of vortex pinning in single crystals of the isovalently substituted iron-based superconductor $\mathrm{BaFe}_{2}\left(\mathrm{As}_{1-x} \mathrm{P}_{x}\right)_{2}$, a material that qualifies as an archetypical clean superconductor, containing only sparse strong pointlike pins [in the sense of C. J. van der Beek et al., Phys. Rev. B 66, 024523 (2002)]. Widely varying critical current values for nominally similar compositions show that flux pinning is of extrinsic origin. Vortex configurations, imaged using the Bitter decoration method, show less density fluctuations than those previously observed in charge-doped $\mathrm{Ba}\left(\mathrm{Fe}_{1-x} \mathrm{Co}_{x}\right)_{2} \mathrm{As}_{2}$ single crystals. Analysis reveals that the pinning force and energy distributions depend on the $\mathrm{P}$ content $x$. However, they are always much narrower than in $\mathrm{Ba}\left(\mathrm{Fe}_{1-x} \mathrm{Co}_{x}\right)_{2} \mathrm{As}_{2}$, a result that is attributed to the weaker temperature dependence of the superfluid density on approaching $T_{c}$ in $\mathrm{BaFe}_{2}\left(\mathrm{As}_{1-x} \mathrm{P}_{x}\right)_{2}$. Critical current density measurements and pinning force distributions independently yield a mean distance between effective pinning centers $\overline{\mathcal{L}} \sim 90 \mathrm{~nm}$, increasing with increasing $\mathrm{P}$ content $x$. This evolution can be understood as being the consequence of the $\mathrm{P}$ dependence of the London penetration depth. Further salient features are a wide vortex free "Meissner belt", observed at the edge of overdoped crystals, and characteristic chainlike vortex arrangements, observed at all levels of $\mathrm{P}$ substitution.
\end{abstract}

DOI: 10.1103/PhysRevB.87.094506

\section{INTRODUCTION}

Recent vortex imaging studies performed on iron pnictide superconductors show evidence for nanoscale inhomogeneity ${ }^{1,2}$ being at the origin of the low-field critical current density and the highly disordered vortex structures in these materials. ${ }^{3-12}$ Notably, in $\mathrm{Ba}\left(\mathrm{Fe}_{1-x} \mathrm{Co}_{x}\right)_{2} \mathrm{As}_{2}$, the critical current density $j_{c}$ and vortex distributions imaged by Bitter decoration could be consistently analyzed, provided that spatial heterogeneity, on a scale of several dozen $\mathrm{nm}$, both of the critical temperature $T_{c}$ and the vortex line energy $\varepsilon_{0}$, is taken to be responsible for flux pinning. ${ }^{2}$ At higher magnetic fields, of the order of several tenths of a tesla, nanoscale heterogeneities are inefficient in pinning flux lines. ${ }^{1,13,14}$ The critical current density is then most likely determined ${ }^{13}$ by the scattering of quasiparticles in the vortex cores associated with the presence of atomic-size defects in the crystal, ${ }^{15,16}$ leading to weak collective pinning. ${ }^{16,17}$ A good candidate for these defects is the dopant atoms themselves. ${ }^{1,2,13,14}$ The nature of
PACS number(s): 74.25.Sv, 74.25.Wx, 74.62.En, 74.70.Xa the dopant atoms is essential for this mechanism: Charged defects lead to different scattering than uncharged defects. ${ }^{13}$ This weak collective pinning contribution to the critical current density manifests itself as a plateau-like behavior in a $j_{c}(B)$ plot. It is present in all charge-doped iron-based superconductors, as well as in $\mathrm{Ba}\left(\mathrm{Fe}_{1-x} \mathrm{Ru}_{x}\right)_{2} \mathrm{As}_{2} \cdot{ }^{18}$

On the other hand, in isovalently substituted $\mathrm{BaFe}_{2}\left(\mathrm{As}_{1-x} \mathrm{P}_{x}\right)_{2}$ there is no indication of weak collective pinning, ${ }^{13}$ which qualifies the material as "clean" with respect to charge-doped iron-based superconductors. Given recent claims $^{11,12}$ that in certain iron-based superconductors, the vortex configuration is more ordered than what was hitherto observed, ${ }^{3-10}$ it is interesting to see whether the absence of weak collective pinning has any impact on the spatial configuration of vortices. From a magnetic force microscopy (MFM) study at magnetic fields up to 100 Oe, Yang et al. claim that vortex configurations in hole-doped single-crystalline $\mathrm{Ba}_{1-x} \mathrm{~K}_{x} \mathrm{Fe}_{2} \mathrm{As}_{2}$ (with $x=0.28$ and $x=0.4$ ) are more ordered than in, e.g., $\mathrm{Ba}\left(\mathrm{Fe}_{1-x} \mathrm{Co}_{x}\right)_{2} \mathrm{As}_{2} .{ }^{2}$ Using an analysis 
method similar to that of Ref. 2, they report pinning forces that are one order of magnitude smaller on average. They also claim the observation of local triangular vortex order in the optimally doped material, even though closer scrutiny (see Sec. III E below) reveals the vortex ensembles to be no more ordered than those in $\mathrm{Ba}\left(\mathrm{Fe}_{0.925} \mathrm{Co}_{0.075}\right)_{2} \mathrm{As}_{2} .{ }^{2}$ Finally, the authors ${ }^{11}$ report on the observation of remarkable vortex chains, both in underdoped $(x=0.28)$ and optimally doped ( $x=0.4) \mathrm{Ba}_{1-x} \mathrm{~K}_{x} \mathrm{Fe}_{2} \mathrm{As}_{2}$. The presence of these is attributed to vortex pinning by the twin boundaries arising from the orthorhombic structure, at least in the underdoped material. Furthermore, neutron scattering experiments on the vortex lattice in isovalently substituted $\mathrm{BaFe}_{2}\left(\mathrm{As}_{1-x} \mathrm{P}_{x}\right)_{2}$ were performed at $T=2 \mathrm{~K}$ by Kawano-Furukawa et al. ${ }^{12}$ No vortex Bragg peaks were found for the optimally substituted compound. However, after annealing the samples at $500^{\circ} \mathrm{C}$, a distorted triangular vortex lattice was observed; this became more ordered as the applied magnetic field was increased from 0.7 to $7 \mathrm{~T} .{ }^{12}$ These results suggest that the disorder responsible for pinning in $\mathrm{BaFe}_{2}\left(\mathrm{As}_{1-x} \mathrm{P}_{x}\right)_{2}$ is extrinsic in nature.

In this work we present and analyze sustainable current density measurements, magneto-optical imaging, and Bitter decoration experiments performed on $\mathrm{BaFe}_{2}\left(\mathrm{As}_{1-x} \mathrm{P}_{x}\right)_{2}$ single crystals with different $x$. In contrast to some reports, ${ }^{19}$ we find strong pinning, presumably by nanoscale heterogeneity, as the only observed pinning contribution in fields up to $5 \mathrm{~T}$. As in $\mathrm{Ba}\left(\mathrm{Fe}_{1-x} \mathrm{Co}_{x}\right)_{2} \mathrm{As}_{2}$ and other materials, ${ }^{6}$ there is no evidence for any extended triangular order in the vortex ensemble; thus, the strong pinning contribution in itself suffices to generate the extreme disorder of the vortex ensemble. The spatial configuration of vortices in isovalently substituted $\mathrm{BaFe}_{2}\left(\mathrm{As}_{1-x} \mathrm{P}_{x}\right)_{2}$ does not present large vortex density fluctuations such as observed in charge-doped $\mathrm{Ba}\left(\mathrm{Fe}_{1-x} \mathrm{Co}_{x}\right)_{2} \mathrm{As}_{2}$ single crystals, ${ }^{2}$ a fact that is attributed to the different temperature dependencies of the superfluid density in the two materials. The quantitative analysis of the vortex configurations in terms of the pinning energy confirms that pinning disorder is somewhat less effective in $\mathrm{BaFe}_{2}\left(\mathrm{As}_{1-x} \mathrm{P}_{x}\right)_{2}$ than in $\mathrm{Ba}\left(\mathrm{Fe}_{1-x} \mathrm{Co}_{x}\right)_{2} \mathrm{As}_{2}$, and that it depends on the $\mathrm{P}$ content $x$. Analysis of the pinning energies, the pinning forces, and the critical current density as function of $\mathrm{P}$ content yields consistent estimates of the effective pin density. This clearly decreases upon increasing the $\mathrm{P}$ content, a behavior that tracks the composition dependence of the scattering rate in the normal state. The main features of our results can be understood in terms of the composition dependence of the vortex line energy, implying that local variations of the superfluid density are a good candidate for the origin of the vortex pinning. The variation of the pin density as well as the variation of the sustainable current density with composition argues against any possible spatially phase-separated superconducting and antiferromagnetic states of the material as being at the origin of pinning.

\section{EXPERIMENTAL DETAILS}

Experiments have been performed on $\mathrm{BaFe}_{2}\left(\mathrm{As}_{1-x} \mathrm{P}_{x}\right)_{2}$ single crystals grown by the self-flux method, ${ }^{20}$ and characterized using energy-dispersive $\mathrm{x}$-ray spectroscopy (EDX) and EDX mapping in a scanning electron microscope (SEM). Crystals with manifest chemical heterogeneity were discarded from further study. The crystals described below present no impurity phases, within the experimental limits of accuracy $\lesssim 1 \%$.

Magnetic flux penetration in crystals with different substitution levels $(x=0.27-0.49)$ was characterized using the magneto-optical imaging (MOI) method ${ }^{1,14,21}$ before further experiments. The MOI technique notably allows one to discard samples with macroscopic defects, and also to extract calibrated flux density profiles. The sustainable current density $j_{s}$ for magnetic fields up to $\mu_{0} H_{a}=500 \mathrm{G}(50 \mathrm{mT})$ was obtained from the gradient of the local magnetic flux density $B$ perpendicular to the crystal surface, using the Bean model. ${ }^{22,23}$ Given the thickness-to-width ratio of these crystals, $d / w \sim 0.25$, one has $\mu_{0} j_{s} \sim 3 d B / d x .^{24}$ The crystal inhomogeneity, and notably the local distribution of $T_{c}$, was characterized using the differential magneto-optical (DMO) method. ${ }^{14,25}$ Measurements in higher magnetic fields were performed using micron-sized Hall probe arrays ${ }^{26}$ tailored in a pseudomorphic GaAlAs/GaAs heterostructure, as well as using a superconducting quantum interference device (SQUID) based magnetometer.

The vortex ensembles in several crystals, of substitution levels $x=0.33,0.36$, and 0.49 , were imaged using the Bitter decoration method $^{2,27}$ at an applied field $\mu_{0} H_{a}=20 \mathrm{G}(2 \mathrm{mT})$ (see Sec. III E). In what follows, individual crystals will be identified as ( $x=$ substitution level, sample number).

\section{RESULTS}

\section{A. Spatial variation of the critical temperature $\boldsymbol{T}_{\boldsymbol{c}}$}

Figures 1(a) and 1(b) present DMO images of the exclusion of an applied field $\mu_{0} H_{a}=1 \mathrm{G}(0.1 \mathrm{mT})$ as one crosses the superconducting to normal transition of $\mathrm{BaFe}_{2}\left(\mathrm{As}_{1-x} \mathrm{P}_{x}\right)_{2}$ single crystals $x=0.27$ No. 2 and $x=0.49$ No. 1 . These images reveal that $T_{c}$ is spatially heterogeneous. While inhomogeneity is especially pronounced in the underdoped samples, see crystal $x=0.27$ No. 2 , it is also observed in the overdoped crystals. A link between the heterogeneity observed in underdoped samples and a possible phase separation between superconducting and spin density wave (SDW) antiferromagnetic phases, and/or that of orthorhombic and tetragonal structural domains, is therefore not obvious.

The $T_{c}$ heterogeneity is quantified by the local transmittivity $\mathcal{T}_{H}=\left[I(\mathbf{r}, T)-I\left(\mathbf{r}, T \ll T_{c}\right)\right] /$ $\left[I\left(\mathbf{r}, T \gg T_{c}\right)-I\left(\mathbf{r}, T \ll T_{c}\right)\right]$, extracted from the luminous intensity $I(\mathbf{r}, T)$ in the DMO images of Fig. 1. $\mathcal{T}_{H}(T)$ is presented in Fig. 2(a) for crystal $x=0.27$ No. 2. The width of the superconducting transition obtained for the various crystals is presented in Fig. 2(b), where the error bar indicates the spread of $T_{c}$ in a given crystal, and the data points give the temperature where $50 \%$ of the crystal has become superconducting.

\section{B. Sustainable current density $\boldsymbol{j}_{s}$}

Figure 3 presents MOI of the magnetic flux penetration (after zero-field cooling) into superconducting $\mathrm{BaFe}_{2}\left(\mathrm{As}_{1-x} \mathrm{P}_{x}\right)_{2}$ single crystals $x=0.33$ No. 1 and $x=0.36$ No. 2, respectively. The former crystal is characterized by very weak bulk pinning and, as a result, a large influence of geometrical $^{28,29}$ and surface barriers. ${ }^{30}$ The influence of the 
(a) $x=0.27$ \#2

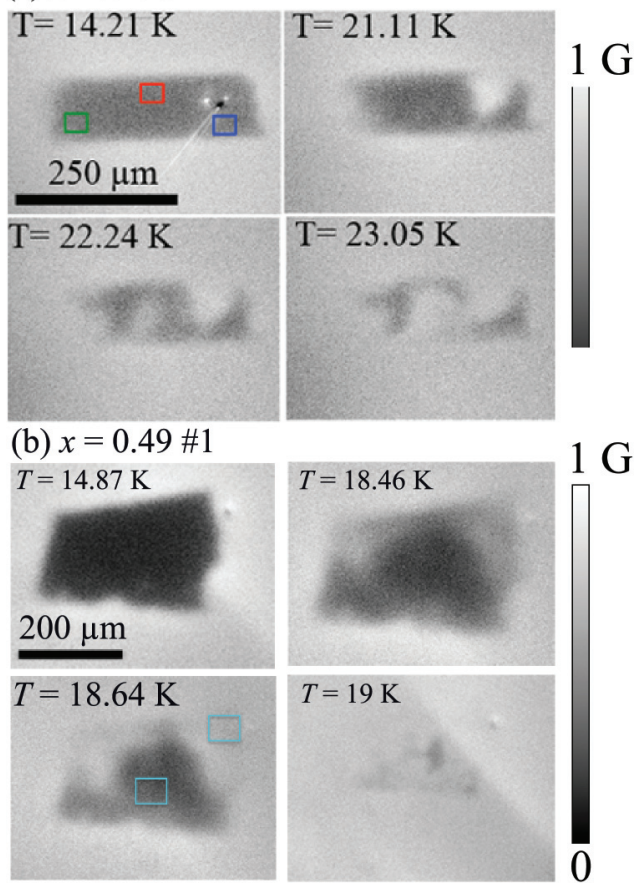

FIG. 1. (Color online) Differential magneto-optical images of the screening of a magnetic field $\mu_{0} H_{a}=1 \mathrm{G}(0.1 \mathrm{mT})$ by $\mathrm{BaFe}_{2}\left(\mathrm{As}_{1-x} \mathrm{P}_{x}\right)_{2}$ single crystals (a) $x=0.27$ No. 2 , and (b) $x=0.49$ No. 1. The intensity is proportional to the local magnetic flux density $B(\mathbf{r})$. Thus, black areas are regions of excluded flux, while the light areas surrounding the crystal are traversed by the applied magnetic field. Rectangular frames in (a) indicate the regions where the transmittivity data of Fig. 2(b) are determined. The frames in (b) denote the areas where the decoration images of Figs. 11(b) and 11(d) were obtained.

surface screening current leads to an inhomogeneous flux density distribution, as presented in Figure 3(a). In contrast, crystal $x=0.36$ No. 2 shows regular flux penetration, in accordance with the Bean critical state model. The influence of a surface barrier, present for both flux entry and flux exit, is also revealed by Hall probe array measurements.

The flux density profiles across the same crystals, depicted in Figs. 4(a)-4(b) and 4(c)-4(d), respectively, were extracted from the calibrated luminous intensities of the magneto-optical images in Fig. 3. One sees that even for the same or comparable doping levels, very different flux density profiles can be obtained after zero-field cooling. Figures 4(c) and 4(d) show the Bean-like penetration of the magnetic flux inside crystal $x=0.36$ No. 2 , with no clear influence of a surface barrier, while the flux profiles for crystal $x=0.33$ No. 1 in Figs. 4(a) and 4(b) show, apart from inhomogeneity, a large discontinuity in the magnetic induction at the sample edge, characteristic of a surface barrier. Given the very different behavior for nearly the same sample composition, the origin of the bulk critical current density in $\mathrm{BaFe}_{2}\left(\mathrm{As}_{1-x} \mathrm{P}_{x}\right)_{2}$ is most likely extrinsic. This is supported by the temperature dependence of the sustainable current density $j_{s}(T, B=30 \mathrm{mT})$ of the studied samples, shown in Fig. 5. The absolute value of $j_{s}(T)$ is widely dispersed, even for crystals with the same doping level.

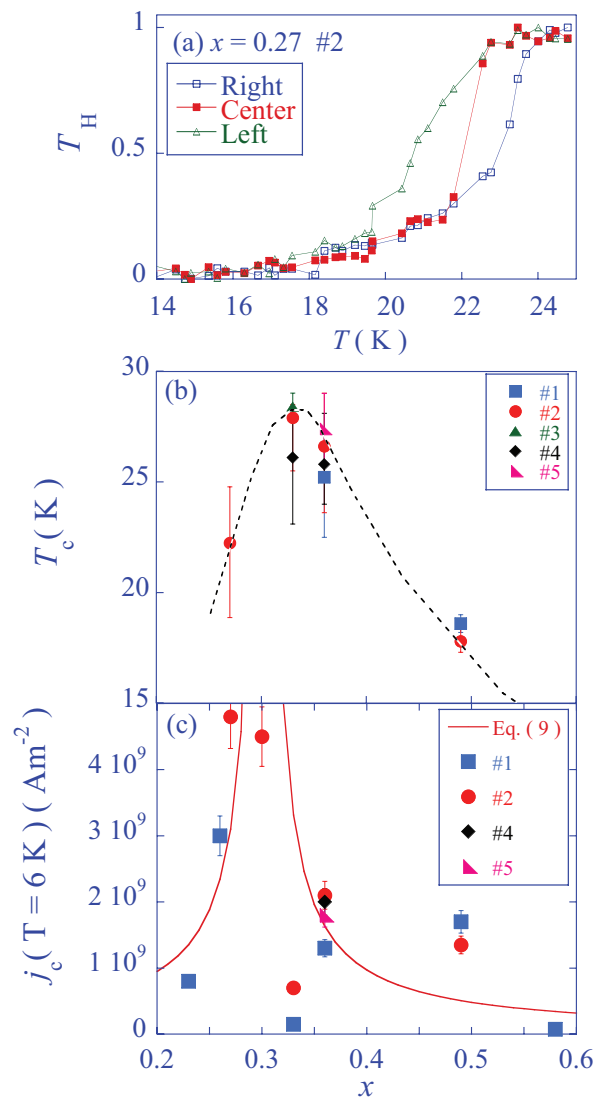

FIG. 2. (Color online) (a) $\mathcal{T}_{H}$ measured on the three regions of crystal $x=0.27$ No. 2 indicated in Fig. 1. (b) Transition temperature $T_{c}$ versus $\mathrm{P}$ content $x$. The error bars indicate the local spread of $T_{c}$ inside a given crystal. For each $x$, the numbering 1,2 , etc., denotes different crystals from the same batch. (c) Dependence of the lowtemperature $(T=6 \mathrm{~K})$ critical current density on $\mathrm{P}$ content $x$. The drawn line shows the evolution of the critical current due to spatial variations of the dopant atom density (on a scale of $\delta z \sim 100 \mathrm{~nm}$ and with variance $\Delta x \sim 0.3 \%$ ) such as expected from Eq. (9).

In spite of the disparity, the flux pinning mechanism in all crystals is the same. Figure 6 shows hysteresis loops of the local gradient of the magnetic induction $d B / d x$ in fields of up to $2 \mathrm{~T}$, obtained on crystals of different composition using the Hall probe array magnetometry technique. ${ }^{26}$ The hysteresis loops were measured at $6 \mathrm{~K}$, at which flux creep has only a moderate influence. For all crystals, of all investigated substitution levels, one has the ubiquitous central peak at zero field, believed to be due to strong pinning by nm-scale disorder. ${ }^{14}$ The magnetic field dependence of the sustainable current density $j_{s}(B)$ was obtained from the value of $d B / d x$ at given $B$. Figure 7 shows $j_{s}(B)$ for the optimally substituted single crystal $x=0.36$ No. 2 at different temperatures. The $j_{s}(B)$ curve at the lowest $T$ is representative of the fielddependent critical current density $j_{c}(B)$. The critical current density is characterized by a low-field plateau, followed by a $j_{c} \propto B^{-1 / 2}$ decrease at higher fields, behavior that is typical of strong flux pinning by sparse pointlike defects. ${ }^{13,14,31}$ The contribution to $j_{c}(B)$ due to weak collective pinning of the vortex lines by atomic-sized point pins, observed in all charge-doped iron-based superconductors as well as 
(a) $x=0.33 \# 1$

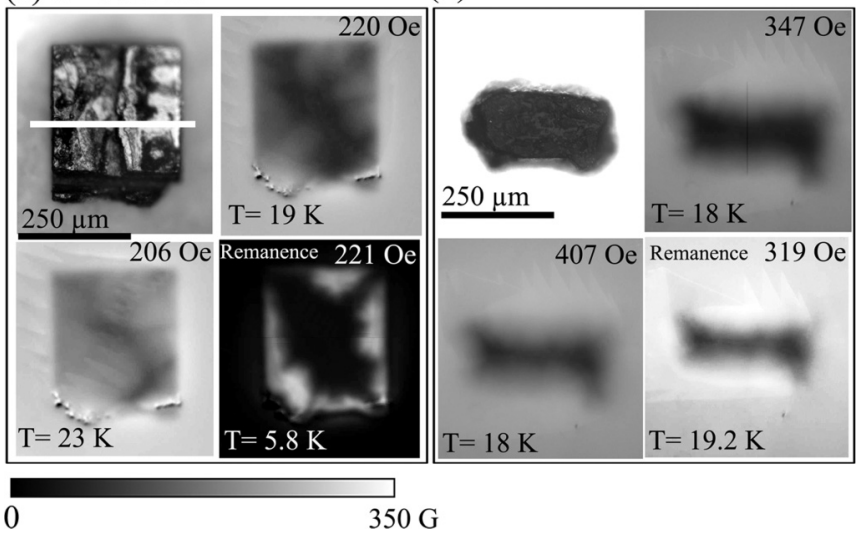

FIG. 3. Magnetic flux density distribution in $\mathrm{BaFe}_{2}\left(\mathrm{As}_{1-x} \mathrm{P}_{x}\right)_{2}$ single crystals $x=0.33$ No. 1 (a), and $x=0.36$ No. 2 (b), after zero-field cooling to the indicated temperatures and the application of different magnetic fields. The top left panel of each subfigure shows the respective crystal; the white lines are those along which the profiles in Fig. 4 are extracted. The intensity in the other panels reflects the local flux density $B(\mathbf{r})$. The bottom right-hand panel of (a) shows the trapped flux distribution in crystal $x=0.33$ No. 1 , after application and removal of $\mu_{0} H_{a}=22.1 \mathrm{mT}$.

in $\mathrm{Ba}\left(\mathrm{Fe}_{1-x} \mathrm{Ru}_{x}\right)_{2} \mathrm{As}_{2}$, is clearly absent in Fig. 7. The $\mathrm{BaFe}_{2}\left(\mathrm{As}_{1-x} \mathrm{P}_{x}\right)_{2}$ system can therefore be seen as a typical strongly pinning superconductor, in the sense that only large but sparse extrinsic pointlike pins contribute to flux pinning. ${ }^{31}$ At larger temperatures and fields, $j_{s}(B)$ decreases faster than $B^{-1 / 2}$, an effect attributed to flux creep.

Measurements carried out to larger fields using a SQUID magnetometer (Fig. 8) show that, contrary to the data presented in Ref. 19, the sustainable current density continues its

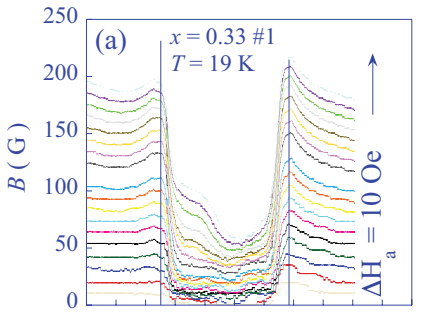

(b)

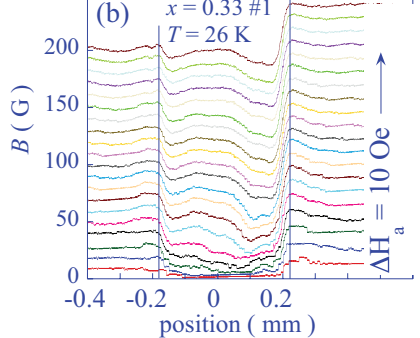

FIG. 4. (Color online) Magnetic flux density profiles in $\mathrm{BaFe}_{2}\left(\mathrm{As}_{1-x} \mathrm{P}_{x}\right)_{2}$ crystal $x=0.33$ No. 1 , at $T=19$ and $26 \mathrm{~K}$ [(a) and (b)], and crystal $x=0.36$ No. 2, at $T=19$ and $22.2 \mathrm{~K} \mathrm{[(c)} \mathrm{and}$ (d)], after zero-field cooling and application of the magnetic field in successive steps $\Delta H_{a}$. The Bean-like profiles in (c) and (d) are obtained from the MOI images of Fig. 3(b). The profiles in (a) and (b) are influenced by a surface barrier and correspond to the crystal of Fig. 3(a).

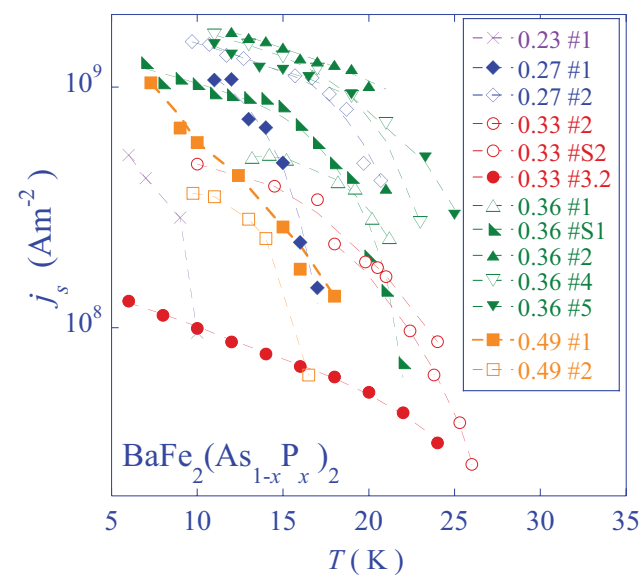

FIG. 5. (Color online) Temperature dependence of the sustainable current density $j_{s}(T, B=30 \mathrm{mT})$, determined from flux density profiles obtained from MOI images, for a variety of crystals with substitution levels $0.23 \leqslant x \leqslant 0.49$. Samples denoted "S" concern a limited area of the sample with the same number. Dashed lines are guides to the eye.

monotonous decrease as a function of magnetic field - there is no "fishtail" or "second-peak" effect ${ }^{14}$ up to $\mu_{0} H_{a}=5 \mathrm{~T}$. The peak effect being usually associated with a weak collective pinning contribution to the critical current, we surmise that in the field range of interest the strong pinning mechanism is the only one at play.

\section{Quantitative effect of flux creep}

The influence of flux creep is assessed from relaxation measurements of the local flux density using the Hall probe magnetometry technique. Typical examples, shown in Fig. 9(a), show that the creep rate $S \equiv d \ln (d B / d x) / d \ln t$ typically amounts to a few percent. Nevertheless, $j_{s}$ is
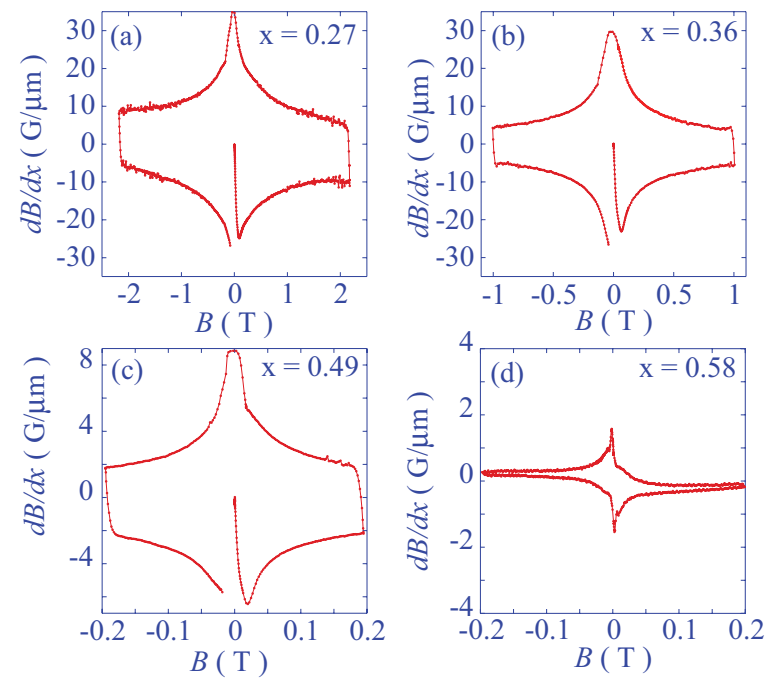

FIG. 6. (Color online) Hysteresis loops of the spatial gradient $d B / d x$ of the local magnetic induction on the surface of $\mathrm{BaFe}_{2}\left(\mathrm{As}_{1-x} \mathrm{P}_{x}\right)_{2}$ single crystals of different substitution levels $0.27 \leqslant x \leqslant 0.58$, measured using the Hall probe magnetometry technique (Ref. 26), at $T=6 \mathrm{~K}$. 


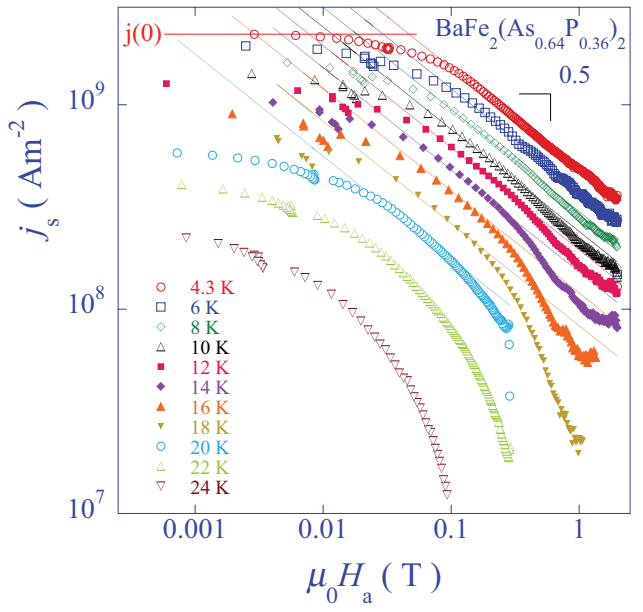

FIG. 7. (Color online) Magnetic field dependence of the sustainable current density $j_{s}$ for crystal $x=0.36$ No. 2, obtained from the local magnetic induction gradient as measured using the Hall probe magnetometry method (Ref. 26).

significantly affected by creep, such that it is determined as the solution of the relation $U\left(j_{s}\right)=k_{B} T \ln \left[\left(t_{0}+t\right) / \tau\right]$, rather than by the critical current density $j_{c} .{ }^{32,33}$ Here, $t_{0}$ is a time describing transient effects at the onset of relaxation, and $\tau$ is a normalization time related to the sample inductance. ${ }^{32,33}$ The dependence of the flux creep barrier $U(j)$ on current density $j$ can be extracted using various methods, including those of Maley et al. ${ }^{34}$ and Abulafia et al. ${ }^{35,36}$ Applying the latter, we find [see Fig. 9(b)] that the creep barrier in optimally substituted $\mathrm{BaFe}_{2}\left(\mathrm{As}_{1-x} \mathrm{P}_{x}\right)_{2}$ follows

$$
U(j)=U_{c}\left(\frac{j_{c}}{j}\right)^{\mu},
$$

with values of the exponent $\mu \sim 1.5-2$. Therefore, the time and temperature dependence of the sustainable screening current

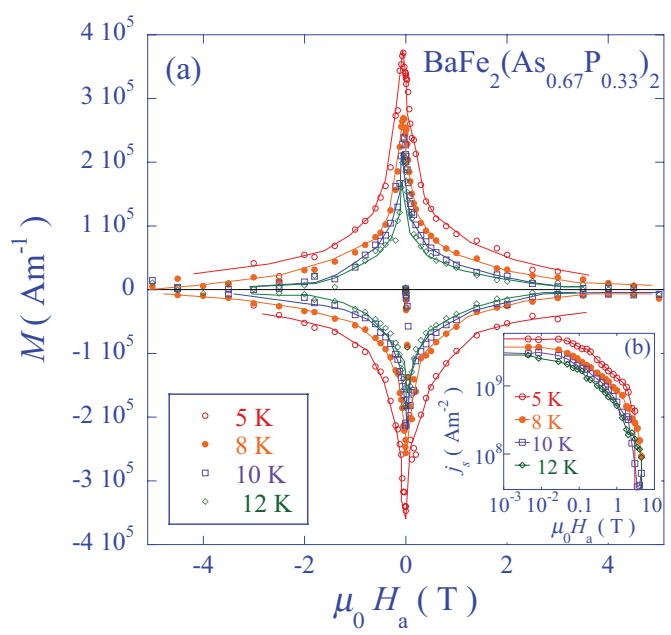

FIG. 8. (Color online) (a) Magnetic hysteresis loops measured on $\mathrm{BaFe}_{2}\left(\mathrm{As}_{1-x} \mathrm{P}_{x}\right)_{2}$ crystal $x=0.33$ No. 2 using a SQUID magnetometer. (b) Sustainable screening current $j_{s}$ as function of the applied field $\mu_{0} H_{a}$ (in teslas).
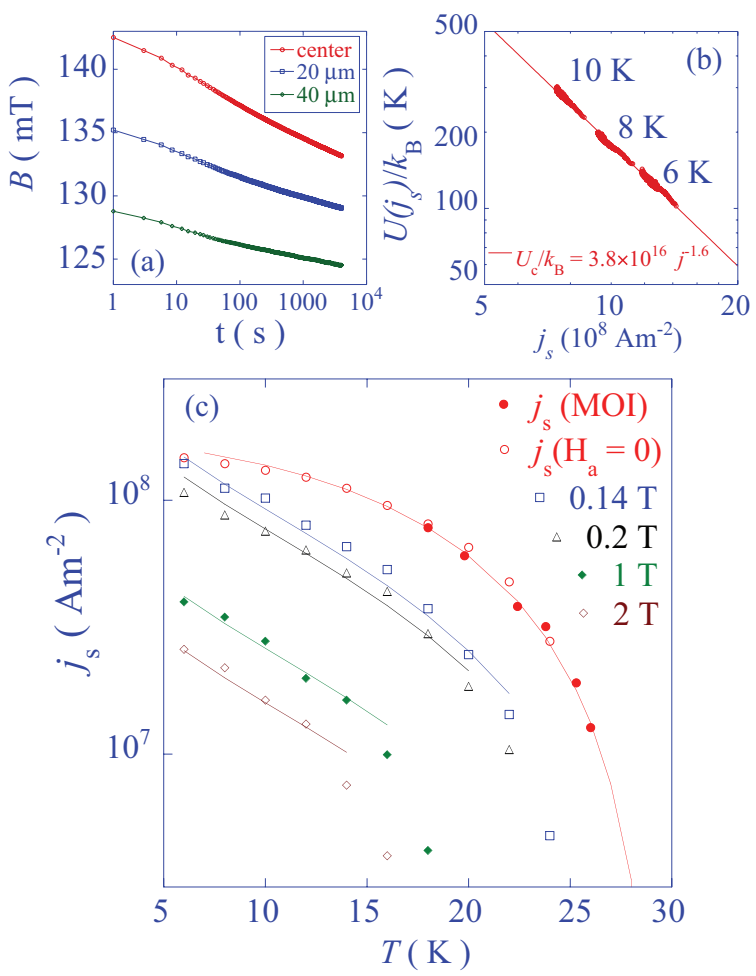

FIG. 9. (Color online) Magnetic relaxation in $\mathrm{BaFe}_{2}\left(\mathrm{As}_{1-x} \mathrm{P}_{x}\right)_{2}$ crystal $x=0.33$ No. 2. (a) Relaxation of the magnetic flux density at three positions on the crystal surface-the center, and positions 20 and $40 \mu \mathrm{m}$ from the center. Data were taken at $10 \mathrm{~K}$, after field cooling in $0.2 \mathrm{~T}$ and subsequently removing the field. (b) Experimental flux-creep activation barrier $U$ versus $j_{s}$, as obtained using the method outlined in Refs. 35 and 36. (c) Temperature dependence of the sustainable current density in zero applied field, and applied fields of $0.14,0.2,1$, and $2 \mathrm{~T}$. Measurements using the Hall probe array technique (open symbols) were obtained from hysteresis loops such as shown in Fig. 6, while data from MOI are obtained from the flux-profile gradient. The drawn line through the $j_{s}\left(H_{a}=0\right)$ data corresponds to the expected $T$ dependence of the depairing current; the drawn lines for higher fields are obtained from this by taking the field dependence of the critical current (3), (4) into account, and by correcting for flux creep using Eq. (2).

density is described by

$$
j_{s}=j_{c}\left[\frac{k_{B} T}{U_{c}} \ln \left(\frac{t+t_{0}}{\tau}\right)\right]^{-1 / \mu} .
$$

The impact of flux creep on the temperature dependence of the sustainable current is depicted in Fig. 9(c), which shows $j_{s}(T)$ curves for crystal $x=0.33$ No. 2, for different $B$. The curve in zero applied field is little affected by creep, and roughly follows the expected temperature dependence of the depairing current, $j(0, T) \sim \varepsilon_{0}(T) / \Phi_{0} \xi(T)\left(\Phi_{0}=h / 2 e\right.$ is the flux quantum). Here, the vortex line energy, $\varepsilon_{0}(T)=$ $\Phi_{0}^{2} / 4 \pi \mu_{0} \lambda_{a b}^{2}$, proportional to the superfluid density $n_{s} \sim$ $\lambda_{a b}^{-2}$, is evaluated using the data for the in-plane penetration depth $\lambda_{a b}(T)$ of Ref. 41, and the coherence length $\xi(T) \sim$ $\xi(0) \sqrt{\left(1+T / T_{c}\right) /\left(1-T / T_{c}\right)}$. The curves for varying applied field can then be well described by taking the creep barrier prefactor $U_{c}(T) \propto \varepsilon_{0}(T), j_{c}(B, T) \propto j(0, T) B^{-1 / 2}, \mu=$ 1.6, and $\ln \left[\left(t+t_{0}\right) / \tau\right]=20 .^{36}$ Therefore, the temperature 
dependence of the screening current in fields larger than $0.1 \mathrm{~T}$ is essentially determined by flux creep.

\section{Extraction of pinning parameters}

We now analyze the $j_{s}(B)$ curves measured at low temperature, representative of the critical current density $j_{c}(B)$, and which bear the hallmarks of strong pinning. These are the plateau at low magnetic field, ${ }^{14,31}$

$$
j_{c}(0)=\frac{f_{p}}{\Phi_{0} \overline{\mathcal{L}}}=\pi^{1 / 2} \frac{f_{p}}{\Phi_{0} \varepsilon_{\lambda}}\left(\frac{U_{p} n_{i}}{\varepsilon_{0}}\right)^{1 / 2} \quad\left(B \ll B^{*}\right),
$$

followed by a power-law decrease as a function of the flux density $B,{ }^{14,31}$ which can be described as

$$
\begin{aligned}
j_{c}(B) & =\frac{f_{p}}{\Phi_{0} \overline{\mathcal{L}}^{2}} \frac{\varepsilon_{\lambda} a_{0}}{\pi} \\
& =\frac{f_{p}}{\Phi_{0} \varepsilon_{\lambda}}\left(\frac{U_{p} n_{i}}{\varepsilon_{0}}\right)\left(\frac{\Phi_{0}}{B}\right)^{1 / 2} \quad\left(B \gg B^{*}\right) .
\end{aligned}
$$

The main parameter, $\overline{\mathcal{L}}=\left(\pi U_{p} n_{i} / \varepsilon_{\lambda} \varepsilon_{0}\right)^{-1 / 2}$, is the average distance between effective defects pinning a single vortex in the low-field limit. ${ }^{31}$ The crossover field $B^{*}$ is that above which the intervortex repulsion limits the number of effective pins per vortex, and $f_{p}$ is the maximum pinning force exerted by a single strong pin. $U_{p} /[J]$ is the pinning energy of a single strong pin, $n_{i}$ is the pin density, $a_{0}=\left(\Phi_{0} / B\right)^{1 / 2}$ is the intervortex distance, and $\varepsilon_{\lambda}=\lambda_{a b} / \lambda_{c}$ is the penetration depth anisotropy.

Equations (3) and (4) show that the pinning force of a single strong pin $f_{p}=\left(\Phi_{0}^{3 / 2} \varepsilon_{\lambda} / \pi\right)\left\{j^{2}(0) /\left[\partial j(B) / \partial B^{-1 / 2}\right]\right\}$ can be obtained from the experimentally measured low-temperature, low-field current density $j_{c}(0)$, and the slope $\partial j_{c}(B) / \partial B^{-1 / 2}$ at intermediate fields. Reserving our attention to the crystals used in the Bitter decoration experiments presented below, we obtain, for an estimated $\varepsilon_{\lambda}=0.15,{ }^{37} f_{p} \approx 8 \times 10^{-13} \mathrm{~N}$ for both crystals $(x=0.36$ No. 2$)$ and ( $x=0.49$ No. 1$)$. This value is twice larger than that measured in $\mathrm{Ba}\left(\mathrm{Fe}_{1-x} \mathrm{Co}_{x}\right)_{2} \mathrm{As}_{2} .{ }^{2} \mathrm{An}$ evaluation at the highest measurement temperature of $0.8 T_{c}$ yields $f_{p}=2 \times 10^{-14} \mathrm{~N}$; however, this value is likely to be overestimated due to creep. Similarly, one can extract the length $\overline{\mathcal{L}}=f_{p} / \Phi_{0} j_{c}(0)$. Figure 10 shows that $\overline{\mathcal{L}}$ is of the order of several dozen to hundreds of $\mathrm{nm}$, in accordance with the strong pinning hypothesis. Moreover, the distance between effective pins clearly increases as a function of P content $x$.

\section{E. Vortex imaging by Bitter decoration}

The Bitter decoration technique ${ }^{2,27}$ was used to image the vortex ensemble in $\mathrm{BaFe}_{2}\left(\mathrm{As}_{1-x} \mathrm{P}_{x}\right)_{2}$ single crystals with three different substitution levels, $x=0.33, x=0.36$, and $x=0.49$. The experiments were realized under field-cooled (FC) conditions, with a field $\mu_{0} H_{a}=20 \mathrm{G}(2 \mathrm{mT})$ applied parallel to the $c$ axis of the crystals. The decoration experiment for the crystal with $x=0.33$ was not successful, presumably due to the large value of the penetration depth. ${ }^{38}$

The vortex configurations shown in Fig. 11, for crystals $x=0.36$ No. 2 and $x=0.49$ No. 1 , are representative of what is observed over the entire specimens. From the decoration images, we obtain, for both crystals, the average value of

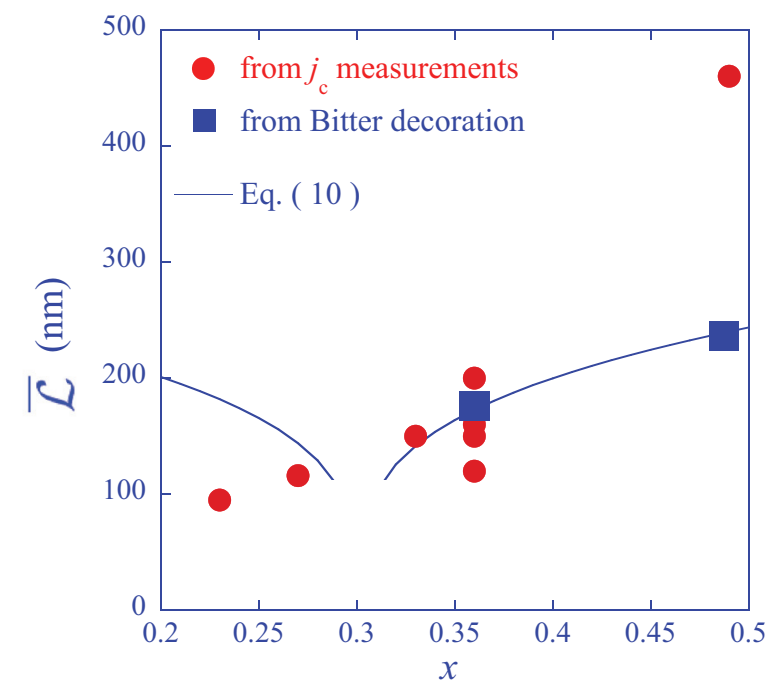

FIG. 10. (Color online) Average distance $\overline{\mathcal{L}}$ between effective pins versus $\mathrm{P}$ content. $\overline{\mathcal{L}}$ is obtained from $j_{s}(B)$ data at $5 \mathrm{~K}$ (red bullets), and from Bitter decoration at $T_{f}=0.87 T_{c}$ (blue squares). The drawn lines show the comparison with Eq. (10), for variations of the dopant atom density $\Delta x=0.3 \%$, on a characteristic length scale $\delta z=100 \mathrm{~nm}$.

the magnetic induction as $B_{\text {int }}=n_{v} \Phi_{0} \approx 19 \mathrm{G}$ (with $n_{v}$ the vortex density). This is $1 \mathrm{G}$ smaller than the applied field during the experiment. Notwithstanding Ref. 39, there is therefore evidence for Meissner exclusion of the magnetic flux. Moreover, the Meissner current manifests itself as a vortex-free "Meissner belt" along the edges of decorated crystal $x=0.49$ No. 1 , as well as near surface steps that appear during preliminary cleavage of the samples, as indicated in Fig. 11. Long vortex chains reminiscent of those observed in Ref. 11 are also observed in the decoration images, for
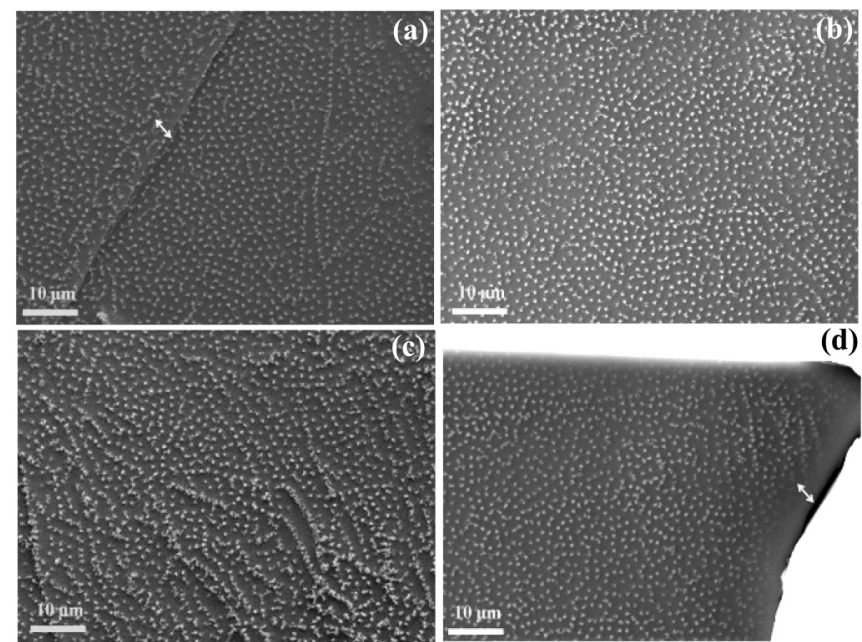

FIG. 11. Bitter decoration images of $\mathrm{BaFe}_{2}\left(\mathrm{As}_{1-x} \mathrm{P}_{x}\right)_{2}$ single crystals for an applied field $\mu_{0} H_{a}=20 \mathrm{G}$ (a), (c) $x=0.36$ No. 2 , and (b) and (d) $x=0.49$ No. 1 . The white arrows indicate the vortex-free Meissner belt observed (a) near a surface step and (d) at the edge of the crystal $x=0.49$ No. 1 . The regions where images (b) and (d) were obtained with respect to the entire crystal are depicted in Fig. 1. 
both investigated $\mathrm{P}$ contents; however, the chains are more pronounced in crystal $x=0.36$ No. 2 .

Figures 12(a) and 12(b) present the respective Delaunay triangulations of the vortex ensembles of Figs. 11(a) and 11(b). Here, blue dots represent vortices with sixfold coordination, while red dots represent vortices with different coordination number. Even if there are some regions with several adjacent sixfold-coordinated vortices, the insets to Figs. 12(c) and 12 (d) reveal that the latter represent less than half of the total (46\% for crystal $x=0.49$ No. 1 , and $43 \%$ for crystal $x=0.36$ No. 2). In fact, for the magnetic field of $20 \mathrm{G}$ under study, the percentage of sixfold-coordinated vortices is the same as in $\mathrm{Ba}\left(\mathrm{Fe}_{0925} \mathrm{Co}_{0.075}\right)_{2} \mathrm{As}_{2}(43 \%)^{40}$ and in $\mathrm{Ba}_{0.6} \mathrm{~K}_{0.4} \mathrm{Fe}_{2} \mathrm{As}_{2}(44 \%) .{ }^{11}$ Moreover, the coordination number histograms have the same width for the three materials, revealing similar disorder of the vortex ensemble. The absence of vortex lattice order is further brought out by the Fourier transforms of the vortex positions shown in the insert to Figs. 12(a) and 12(b). Nevertheless, the spatial distribution of vortices in the two $\mathrm{BaFe}_{2}\left(\mathrm{As}_{1-x} \mathrm{P}_{x}\right)_{2}$ crystals presents smaller density fluctuations than previously observed in the Co-doped material. ${ }^{2}$ Panels (c) and (d) of Fig. 12 show the distributions of nearest-neighbor intervortex distances. These have a mean value $\left|r_{i j}\right|=1 \mu \mathrm{m}$, while the lattice parameter for a triangular perfect lattice of the same density is $a_{\triangle}=1.075 \sqrt{\Phi_{0} / B}=$ $1.12 \mu \mathrm{m}$. This shift is due to the existence of more densely packed vortices, notably in the chainlike structures.

\section{DISCUSSION}

In what follows, we adopt the procedure of Ref. 2 to determine the vortex interaction energy. For this, one needs to know the value of $\lambda_{a b}$ at the temperature $T_{f}$ at which the vortex ensemble is frozen. As in Ref. 2, we use the information that can be obtained from vortex lines situated near surface steps. Such steps may act as barriers, but, due to the circulation of the Meissner current, they also prevent vortex lines from being situated right at their edge. Inserting the height of the surface step in Fig. 11(a), $h=1.3 \mu \mathrm{m}$, and the width of the vortex-free region close to the step, $u=1.2 \mu \mathrm{m}$, in Eq. (1) of Ref. 2, the value of the penetration depth at the freezing temperature is graphically estimated as $\lambda_{a b}\left(T_{f}\right) \approx 700 \mathrm{~nm}$. Using the temperature dependence of $\lambda_{a b}(T)$ from Ref. 41, one obtains the freezing temperature of the vortex ensemble as $T_{f} \approx 0.87 T_{c}$. Thus, even though vortices are frozen at a relatively high reduced temperature, its value is lower than $T_{f} / T_{c}=0.95$ found in $\mathrm{Ba}\left(\mathrm{Fe}_{1-x} \mathrm{Co}_{x}\right)_{2} \mathrm{As}_{2}$.

Using $\lambda_{a b}\left(T_{f}\right)$ and the vortex positions extracted from Fig. 11, the interaction energies of the individual vortices can be calculated as

$$
\mathcal{E}_{\text {int }}^{i}=\sum_{j} 2 \varepsilon_{0} K_{0}\left(\frac{\left|r_{i j}\right|}{\lambda_{a b}}\right) .
$$

Here $K_{0}(x)$ is the lowest-order modified Bessel function, and $\left|r_{i j}\right|$ is the distance from vortex $i$ to vortex $j$. For each vortex $i$, only neighbors $j$ contained within a circle of radius of $10 \lambda_{a b}(T)$ are taken into account. This radius was chosen after verification that vortices situated at a larger distance do not significantly contribute to $\mathcal{E}_{\text {int }}^{i}$.
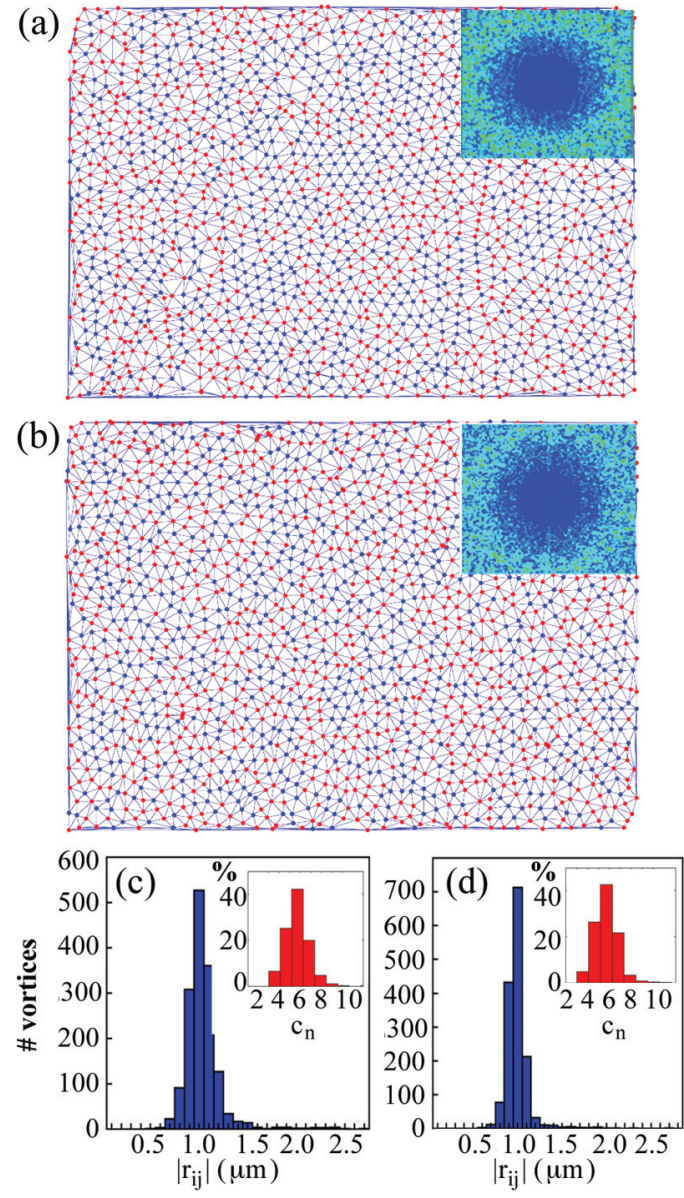

FIG. 12. (Color online) Delaunay triangulation of vortex ensembles observed in $\mathrm{BaFe}_{2}\left(\mathrm{As}_{1-x} \mathrm{P}_{x}\right)_{2}$ single crystals (a) $x=0.36$ No. 2 and (b) $x=0.49$ No. 1 , and presented in Figs. 11(a) and 11(c), respectively. Blue dots represent vortices with sixfold coordination, while red dots represent differently coordinated vortices. The insets show the Fourier transform of the vortex positions. (c) and (d) Nearestneighbor distance distributions for the respective triangulations. The insets present the respective coordination number distributions.

Figures 13(a)-13(d) present the vortex interaction energies as color-coded maps, with the energy scale normalized by $\varepsilon_{0}\left(T_{f}\right)$, as extracted from the images of Figs. 11(a)-11(d), respectively. The maps show a globally homogeneous distribution; however, a number of denser regions exist. Histograms of the interaction energies for the maps (a) to (d) are presented in Figs. 13(e)-13(h). Note that the presence of the chainlike features with a denser vortex arrangement broadens the histograms for $\mathrm{BaFe}_{2}\left(\mathrm{As}_{1-x} \mathrm{P}_{x}\right)_{2}$ single crystal $(x=0.36$ No. 2). Still, the energy distributions are considerably narrower than those found in $\mathrm{Ba}\left(\mathrm{Fe}_{1-x} \mathrm{Co}_{x}\right)_{2} \mathrm{As}_{2} .{ }^{2}$ Furthermore, all distributions are centered about the average $\overline{\mathcal{E}}_{\text {int }} \approx 3.5 \varepsilon_{0}$, which corresponds to the interaction energy value ( $\delta$ peak) of the perfectly triangular Abrikosov lattice for this particular vortex density. Therefore, in contrast to $\mathrm{Ba}\left(\mathrm{Fe}_{1-x} \mathrm{Co}_{x}\right)_{2} \mathrm{As}_{2},{ }^{2}$ no pinning-induced shift of the average value of the energy distribution histogram with respect to the $\delta$-peak value is observed.

The large shift found in Ref. 2 was interpreted in terms of a large average pinning energy in the vicinity of $T_{c}$, which 

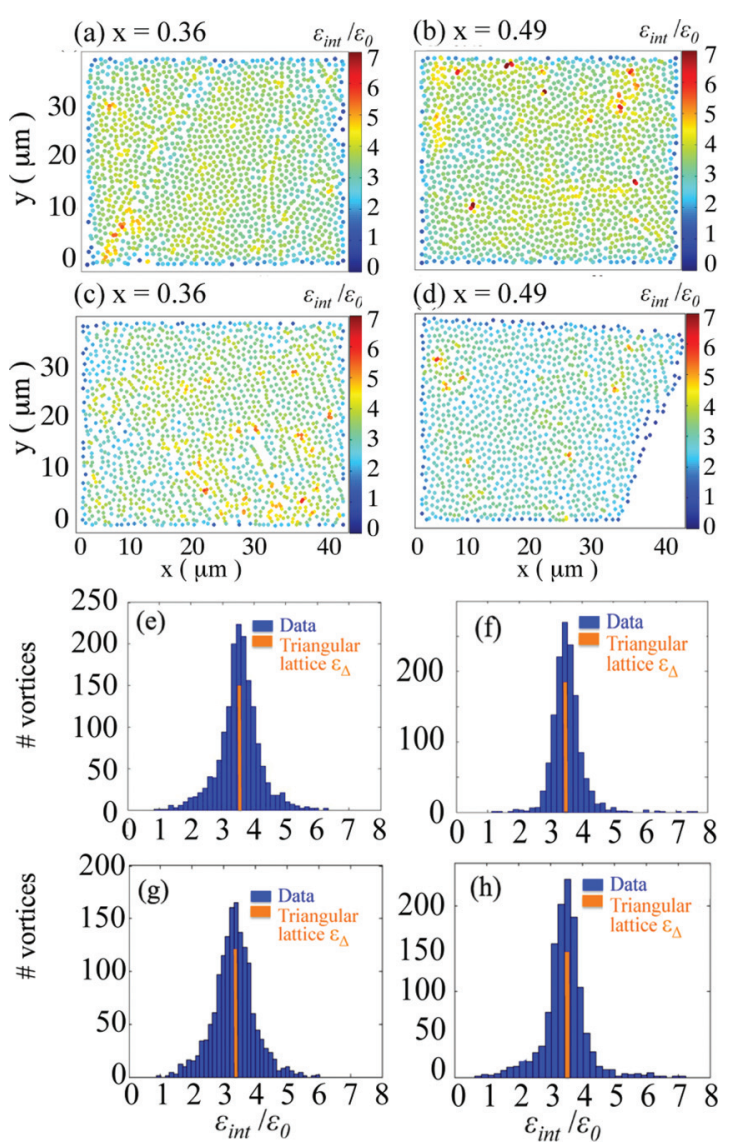

FIG. 13. (Color online) (a)-(d) Color-coded maps of the normalized individual vortex interaction energies calculated from the images of Figs. 11(a)-11(d) using Eq. (5), and represented in the same configuration. Lower panels: Normalized interaction energy distributions for $\mathrm{BaFe}_{2}\left(\mathrm{As}_{1-x} \mathrm{P}_{x}\right)_{2}$ crystals $(x=0.36$ No. 2$)[(\mathrm{e})$ and (g)], and ( $x=0.49$ No. 1) [(f) and (h)]. Vortices located within a distance $10 \lambda_{a b}$ from the map edge are excluded from the histograms. The interaction energy per vortex of the triangular lattice ( $\delta$ function) is represented by the central orange line in each histogram.

can only be the result of $T_{c}$ heterogeneity. The absence of such a shift in isovalently substituted $\mathrm{BaFe}_{2}\left(\mathrm{As}_{1-x} \mathrm{P}_{x}\right)_{2}$ suggests that spatial inhomogeneity of $T_{c}$ is irrelevant for vortex pinning in this material. A probable reason for this is a smoother temperature dependence of the pinning potential. The pinning energy due to spatial variations of the vortex line energy, on a scale $\delta z$ and with variance $\Delta \varepsilon_{0}$, is written $U_{p} \sim \Delta \varepsilon_{0}(T) \delta z \sim\left[\Delta n_{s}(0) / n_{s}(0)\right]\left[\partial \varepsilon_{0}(T) / \partial n_{s}(0)\right] n_{s}(T)+$ $\left[\varepsilon_{0} / n_{s}(0)\right]\left[\partial n_{s}(T) / \partial T_{c}\right] \Delta T_{c}$. At temperatures close to $T_{c}$, the second contribution, due to spatial fluctuations of $T_{c}$, dominates the pinning energy. ${ }^{2}$ However, whereas in materials such as $\mathrm{Ba}\left(\mathrm{Fe}_{1-x} \mathrm{Co}_{x}\right)_{2} \mathrm{As}_{2}$-in which $n_{s}$ is nearly linear in $1-T / T_{c}$ as one approaches $T_{c}$ (see Fig. 14)-this contribution is large, in $\mathrm{BaFe}_{2}\left(\mathrm{As}_{1-x} \mathrm{P}_{x}\right)_{2}$ with a smoother temperature dependence of $n_{s}$ this contribution vanishes. In fact, the $T_{c}$ heterogeneity within the decorated areas of the P-substituted material, such as observed by the DMO technique, does not result in qualitatively different vortex arrangements in different parts of the crystal [see Fig. 1(b)].

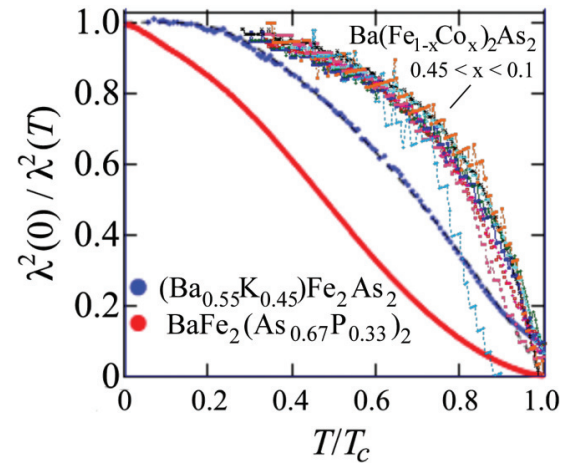

FIG. 14. (Color online) Temperature dependence of the superfluid density, $n_{s}(T) / n_{s}(0) \propto \lambda_{a b}^{2}(0) / \lambda_{a b}^{2}(T)$, for various doping levels $x$ of $\mathrm{Ba}\left(\mathrm{Fe}_{1-x} \mathrm{Co}_{x}\right)_{2} \mathrm{As}_{2}$ (upper curves, small data points), $\mathrm{Ba}_{0.55} \mathrm{~K}_{0.45} \mathrm{Fe}_{2} \mathrm{As}_{2}$ (blue bullets), and $\mathrm{BaFe}_{2}\left(\mathrm{As}_{0.67} \mathrm{P}_{0.33}\right)_{2}$ (red bullets) (both from Ref. 41).

Figure 15 shows maps of the modulus of the pinning force for each individual vortex, calculated from

$$
\mathbf{f}_{i}=\sum_{j} \frac{2 \varepsilon_{0}}{\lambda_{a b}} \frac{\mathbf{r}_{i j}}{\left|\mathbf{r}_{i j}\right|} K_{1}\left(\frac{\left|\mathbf{r}_{i j}\right|}{\lambda_{a b}}\right)
$$

following a procedure similar to that used for the determination of $\mathcal{E}_{\text {int }}^{i}$. Here $K_{1}(x)$ is the first-order modified Bessel function. Since the rendered vortex configurations in Fig. 11 are in a stationary state at the freezing temperature $T_{f}$, the calculated intervortex repulsive force must be balanced by the pinning force. The maps of Figs. 15(a) to 15(d) therefore represent the minimum local pinning force for each vortex, $\min \left(\left|\mathbf{f}_{i}\right|\right)$. The distributions of $\min \left(\left|\mathbf{f}_{i}\right|\right)$ shown in Figs. 15(e) to 15(h) allow one to estimate the average pinning force per vortex and per unit length. We obtain $\left|\overrightarrow{\mathbf{f}}_{i}\right| \sim 3.5 \times 10^{-6} \mathrm{~N} \mathrm{~m}^{-1}$ for crystal $x=0.49$ No. 1 and $\left|\overline{\mathbf{f}}_{i}\right| \sim 4.5 \times 10^{-6} \mathrm{~N} \mathrm{~m}^{-1}$ for crystal $x=$ 0.36 No. 2. These (high temperature) values are comparable to those found in $\mathrm{Ba}\left(\mathrm{Fe}_{1-x} \mathrm{Co}_{x}\right)_{2} \mathrm{As}_{2} .^{2}$

The maps of the local pinning force moduli and the interaction energy shown in Figs. 13 and 15, respectively, are correlated. However, the respective probability distributions are clearly broader for the lower substitution level $x=0.36$. As in Ref. 2, the ratio of the elementary pinning force per pin $f_{p}$, extracted from the $j(B)$ curves in Sec. III A, and the value of $\left|\overline{\mathbf{f}}_{i}\right|$ obtained from Bitter decoration allows one to evaluate an upper bound on $\overline{\mathcal{L}}$ in an independent manner. Using the low-temperature value $f_{p} \sim 8 \times 10^{-13} \mathrm{~N}$ yields $\overline{\mathcal{L}}=180 \mathrm{~nm}$ for crystal $x=0.36$ No. 2 and $\overline{\mathcal{L}}=230 \mathrm{~nm}$ for crystal $x=0.49$ No. 1 . Figure 10 shows that these numbers are consistent with those directly extracted from the sustainable current density measurements.

Several reasons can be invoked to explain the observed enhancement of $\overline{\mathcal{L}}$ with increasing $\mathrm{P}$ content $x$. First is the increase of the superfluid density $n_{s}(x)$ as function of $x .^{38}$ Other possibilities are a decrease of the density $n_{i}$ of pinning centers for larger $x$, and the decrease of the penetration depth anisotropy $\varepsilon_{\lambda}^{-1}$ for higher substitution levels. We first investigate the effect of the increase of the superfluid density with $x$. Assuming that nm-scale fluctuations of the dopant atom density (with variance $\Delta x$ ) are spatially isotropic, their 

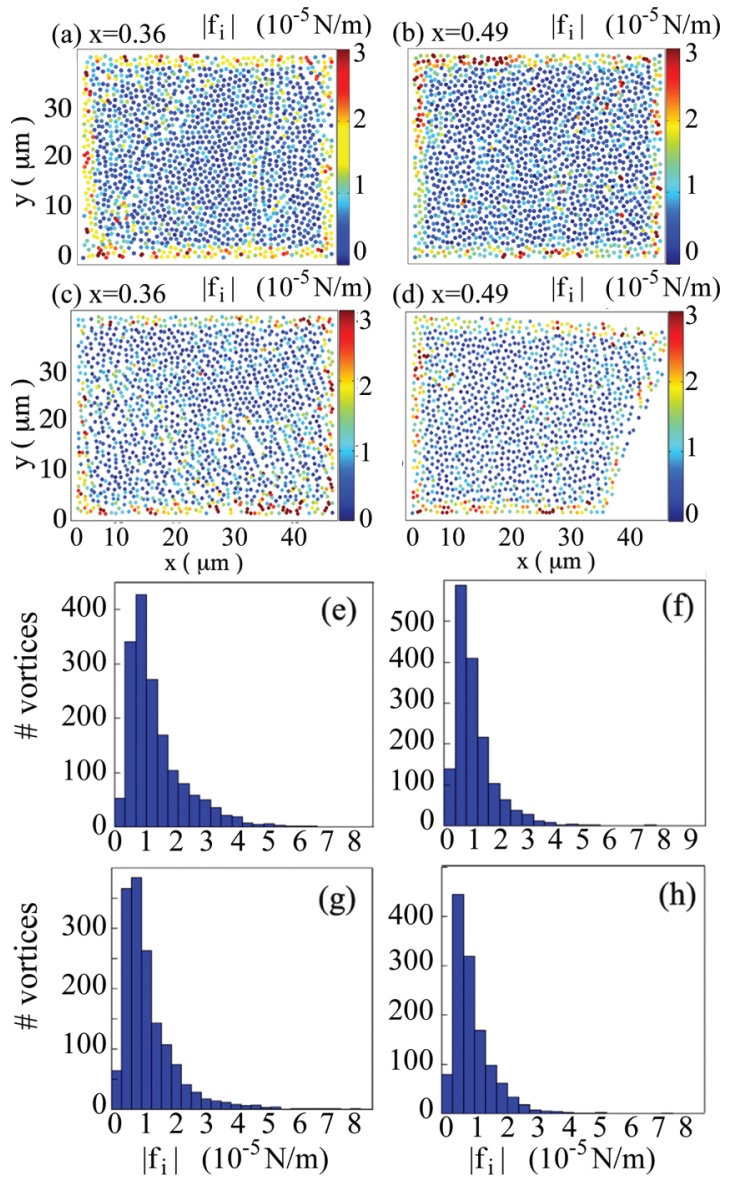

FIG. 15. (Color online) Normalized color-coded maps of the modulus of the pinning force (per unit length), calculated from the images of Fig. 11 using Eq. (6). Lower panels: Pinning force distributions for $\mathrm{BaFe}_{2}\left(\mathrm{As}_{1-x} \mathrm{P}_{x}\right)_{2}$ single crystals ( $x=0.36$ No. 2) [(e) and (g)], and ( $x=0.49$ No. 1) [(f) and (h)]. Again, vortices located within a distance $10 \lambda_{a b}$ from the map edge are excluded from the histograms.

effect on the pinning force

$$
\left\langle f_{p}\right\rangle \sim\left\langle\int_{\delta z} \nabla \varepsilon_{0}(r) d z\right\rangle \sim \Delta \varepsilon_{0}
$$

and the pinning energy $U_{p} \sim \Delta \varepsilon_{0} \delta z$ can be estimated by exploiting the dependence $\lambda_{a b}(x),{ }^{38}$

$$
\left\langle f_{p}\right\rangle \sim \Delta \varepsilon_{0} \sim \frac{\partial \varepsilon_{0}}{\partial \lambda_{a b}} \frac{\partial \lambda_{a b}}{\partial x} \Delta x .
$$

The low-field critical current density (3) becomes

$$
\begin{aligned}
j_{c} & \sim \frac{\pi^{1 / 2} n_{i}}{\Phi_{0} \varepsilon_{\lambda}} \frac{\Delta \varepsilon_{0}^{3 / 2}}{\varepsilon_{0}^{1 / 2}} \\
& =\frac{n_{i}}{\Phi_{0} \varepsilon_{\lambda}}\left(\frac{\pi}{\varepsilon_{0}(x)}\right)^{1 / 2}\left|\frac{\partial \varepsilon_{0}}{\partial \lambda_{a b}} \frac{\partial \lambda_{a b}}{\partial x} \Delta x\right|^{3 / 2},
\end{aligned}
$$

the distance between effective pinning centers

$$
\begin{aligned}
\overline{\mathcal{L}} & \sim \frac{\varepsilon_{\lambda}}{\pi^{1 / 2}}\left(\frac{\varepsilon_{0}}{n_{i} \Delta \varepsilon_{0} \delta z}\right)^{1 / 2} \\
& =\frac{\varepsilon_{\lambda}}{2 \pi^{1 / 2}} \frac{1}{\left(n_{i} \delta z\right)^{1 / 2}}\left|\frac{1}{\varepsilon_{0}} \frac{\partial \varepsilon_{0}}{\partial \lambda_{a b}} \frac{\partial \lambda_{a b}}{\partial x} \Delta x\right|^{-1 / 2} .
\end{aligned}
$$

Assuming that for density fluctuations of the dopant atoms the pin density $n_{i} \sim(\delta z)^{-3}$ scales as the inverse cube of the defect size, these expressions can be directly compared to the dependence of the critical current density on $\mathrm{P}$ content [see Fig. 2(c)], as well as that of $\overline{\mathcal{L}}$ (see Fig. 10). For $\delta z=100 \mathrm{~nm}$ and $\Delta x=0.3 \%$, the qualitative trend with $x$ of both quantities can be reproduced. Thus, the dependence of the penetration depth on $\mathrm{P}$ content accounts, at least in the overdoped regime, for an enhanced probability of encountering larger critical current densities around optimal substitution. The similar dispersion of critical current density and pinning length data for different $x$ around the model estimations (9) and (10) in Figs. 2(c) and 10 would then be due to similar disorder of the $\mathrm{P}$ distribution in the different growth batches, eliminating the need to invoke a decreasing disorder with increasing $x$. Still, the evolution $\overline{\mathcal{L}}(x)$ is reminiscent of that of the normal-state mean-free path, extracted by Shishido et al. from de Haas-van Alphen oscillations of the Landau magnetization. ${ }^{42}$ They reported that the mean-free path for the $\beta$ orbits in $\mathrm{BaFe}_{2}\left(\mathrm{As}_{1-x} \mathrm{P}_{x}\right)_{2}$ single crystals increases from $l \sim 170 \AA$ to $800 \AA$ when $\mathrm{P}$ content varies from $x=0.41$ to 1 . This would imply the presence of structural defects that act as strong pinning centers that are unrelated to spatial composition fluctuations. Last but not least, the increase of $\varepsilon_{\lambda}(x)$, as inferred from the doping-dependent evolution of the Fermi surface ${ }^{42}$ and corresponding to a smaller Fe-pnictogen distance for large $x$, would lead to stiffer vortex lines and to less pinning for higher $\mathrm{P}$ content.

Finally, we address the clear presence of chainlike structures in the Bitter decoration images. The following hypotheses can be suggested to explain the appearance of these chains. The first is the possibility of native heterogeneity of the crystals under study, introduced during growth. The presence of linelike defects, or of linear agglomerates of pointlike defects giving stronger local pinning, will lead to the appearance of vortex alignments or chainlike structures, much as this was found in, e.g., $\mathrm{Bi}_{2} \mathrm{Sr}_{2} \mathrm{CaCu}_{2} \mathrm{O}_{8+\delta} \cdot{ }^{43-47} \mathrm{~A}$ second possibility is that, the vortex images having been obtained after field cooling, and since the $\mathrm{BaFe}_{2}\left(\mathrm{As}_{1-x} \mathrm{P}_{x}\right)_{2}$ material under consideration shows Meissner expulsion, a certain fraction of vortices must exit the material before the vortex ensemble is frozen at $T_{f}$. The chains possibly correspond to flow channels for these exiting vortices, the flux in intermediate areas remaining pinned. ${ }^{48}$ Finally, the multiband character of $\mathrm{BaFe}_{2}\left(\mathrm{As}_{1-x} \mathrm{P}_{x}\right)_{2}$ may be responsible for the occurrence of vortex chains, with the possible existence of an attractive part in the intervortex potential. ${ }^{49}$ We leave these questions open for further work.

\section{SUMMARY AND CONCLUSION}

We have presented an overview of vortex pinning in single crystals of the isovalently substituted iron-based superconductor $\mathrm{BaFe}_{2}\left(\mathrm{As}_{1-x} \mathrm{P}_{x}\right)_{2}$, in which we have attempted to correlate the sustainable screening current density as a function of temperature, field, and doping $x$, with the structural properties of the vortex ensemble. The critical current density in $\mathrm{BaFe}_{2}\left(\mathrm{As}_{1-x} \mathrm{P}_{x}\right)_{2}$ is, overall, very well described by the strong pinning scenario of Ref. 31, which allows one to extract 
elementary pinning forces (of the order of $10^{-13} \mathrm{~N}$ ) and the distance between effective pins. The latter is of the order of $100 \mathrm{~nm}$, and increases as a function of doping level $x$. These values are consistent with those independently obtained by means of the magnetic decoration technique. Contrary to Ref. 19, we find no contribution of weak collective pinning to the sustainable current density, suggesting that $\mathrm{P}$ atoms are not responsible for quasiparticle scattering. ${ }^{13}$ The sustainable current data are affected by flux creep, which prohibits one from drawing definite conclusions concerning the temperature dependence of pinning.

Bitter decoration reveals slightly more ordered vortex ensembles (the number of sixfold-coordinated vortices is slightly higher) than those observed in charge-doped $\mathrm{Ba}\left(\mathrm{Fe}_{1-x} \mathrm{Co}_{x}\right)_{2} \mathrm{As}_{2}$. Also, the interaction energy and pinning force distributions in $\mathrm{BaFe}_{2}\left(\mathrm{As}_{1-x} \mathrm{P}_{x}\right)_{2}$ are much narrower than those in $\mathrm{Ba}\left(\mathrm{Fe}_{1-x} \mathrm{Co}_{x}\right)_{2} \mathrm{As}_{2}$, and are not shifted with respect to the interaction energy of a perfectly triangular vortex lattice with the same density. These observations exclude a role of spatial variations of the critical temperature $T_{c}$ in determining the frozen vortex state obtained upon field cooling. The absence of the weak-collective pinning contribution to the critical current density in $\mathrm{BaFe}_{2}\left(\mathrm{As}_{1-x} \mathrm{P}_{x}\right)_{2}$ means that the strong pinning contribution is what generates the disordered vortex configurations.
The main features of strong vortex pinning in $\mathrm{BaFe}_{2}\left(\mathrm{As}_{1-x} \mathrm{P}_{x}\right)_{2}$, such as the energy and force histograms, the density of effective pins, and the evolution of the critical current density with $\mathrm{P}$ content, can be understood using a model for heterogeneity of the superfluid density on the scale of several dozen to several hundred nanometers, due to an inhomogeneous distribution of the dopant atoms. A small spatial variance $\Delta x$ (of the order of $0.3 \%$ ) suffices to explain the magnitude of $j_{c}$. This model explains why pinning is of extrinsic origin, and why the disorder can be readily annealed. ${ }^{12}$ Even if the $x$ dependence of the critical current density can be well described without any assumption of less disorder in crystals with higher $\mathrm{P}$ content, the reminiscence of the evolution with $\mathrm{P}$ content of the mean distance between effective pinning sites and the normal-state mean-free path ${ }^{42}$ suggests that a second type of pinning center may be at play. This could be areas of enhanced local strain, more prominent at optimal doping, such as those arising from the very different $\mathrm{Fe}-\mathrm{As}$ and Fe-P distances. ${ }^{50}$

\section{ACKNOWLEDGMENTS}

This work was made possible thanks to the support of the ECOS-Sud-MINCyT France-Argentina bilateral program, Grant No. A09E03. Work done in Bariloche was partially funded by PICT 2007-00890 and PICT 2008-294.
${ }^{1}$ C. J. van der Beek, S. Demirdiş, M. Konczykowski, Y. Fasano, N. R. Cejas Bolecek, H. Pastoriza, D. Colson, and F. RullierAlbenque, Physica 407, 1746 (2012).

${ }^{2}$ S. Demirdiş, C. J. van der Beek, Y. Fasano, N. R. Cejas Bolecek, H. Pastoriza, D. Colson, and F. Rullier-Albenque, Phys. Rev. B 84, 094517 (2011).

${ }^{3}$ M. R. Eskildsen, L. Ya. Vinnikov, I. S. Veshchunov, T. M. Artemova, T. D. Blasius, J. M. Densmore, C. D. Dewhurst, N. Ni, A. Kreyssig, S. L. Bud'ko, P. C. Canfield, and A. I. Goldman, Physica C 469, 529 (2009).

${ }^{4}$ M. R. Eskildsen, L. Ya. Vinnikov, T. D. Blasius, I. S. Veshchunov, T. M. Artemova, J. M. Densmore, C. D. Dewhurst, N. Ni, A. Kreyssig, S. L. Bud'ko, P. C. Canfield, and A. I. Goldman, Phys. Rev. B 79, 100501 (2009).

${ }^{5}$ D. S. Inosov, T. Shapoval, V. Neu, U. Wolff, J. S. White, S. Haindl, J. T. Park, D. L. Sun, C. T. Lin, E. M. Forgan, M. S. Viazovska, J. H. Kim, M. Laver, K. Nenkov, O. Khvostikova, S. Kuhnemann, and V. Hinkov, Phys. Rev. B 81, 014513 (2010).

${ }^{6}$ L. Y. Vinnikov, T. M. Artemov, I. S. Veshchunov, N. D. Zhigadlo, J. Karpinski, P. Popovich, D. L. Sund, C. T. Lind, and A. V. Boris, JETP Lett. 90, 299 (2009).

${ }^{7}$ L. Luan, O. M. Auslaender, T. M. Lippman, C. W. Hicks, B. Kalisky, J.-H. Chu, J. G. Analytis, I. R. Fisher, J. R. Kirtley, and K. A. Moler, Phys. Rev. B 81, 100501(R) (2010).

${ }^{8}$ Y. Yin, M. Zech, T. L. Williams, and J. E. Hoffman, Physica C 469, 535 (2009).

${ }^{9}$ B. Kalisky, J. R. Kirtley, J. G. Analytis, J. H. Chu, I. R. Fisher, and K. A. Moler, Phys. Rev. B 83, 064511 (2011).

${ }^{10}$ H. Kawano-Furukawa, C. J. Bowell, J. S. White, J. L. Gavilano, R. W. Heslop, A. S. Cameron, E. M. Forgan, K. Kihou, C. H. Lee,
A. Iyo, H. Eisaki, T. Saito, H. Fukazawa, Y. Kohori, R. Cubitt, C. D. Dewhurst, and M. Zolliker, Phys. Rev. B 84, 024507 (2011).

${ }^{11}$ H. Yang, B. Shen, Z. Wang, L. Shan, C. Ren, and H. H. Wen, Phys. Rev. B 85, 014524 (2012).

${ }^{12}$ H. Kawano-Furukawa et al., in abstract book of ICSM 2012, p. 641.

${ }^{13}$ C. J. van der Beek, M. Konczykowski, S. Kasahara, T. Terashima, R. Okazaki, T. Shibauchi, and Y. Matsuda, Phys. Rev. Lett. 105, 267002 (2010)

${ }^{14}$ C. J. van der Beek, G. Rizza, M. Konczykowski, P. Fertey, I. Monnet, T. Klein, R. Okazaki, M. Ishikado, H. Kito, A. Iyo, H. Eisaki, S. Shamoto, M. E. Tillman, S. L. Bud'ko, P. C. Canfield, T. Shibauchi, and Y. Matsuda, Phys. Rev. B 81, 174517 (2010).

${ }^{15}$ E. V. Thuneberg, J. Kurkijärvi, and D. Rainer, Phys. Rev. Lett. 48, 1853 (1982); Phys. Rev. B 29, 3913 (1984).

${ }^{16}$ G. Blatter, M. V. Feigel'man, V. B. Geshkenbein, A. I. Larkin, and V. M. Vinokur, Rev. Mod. Phys. 66, 1125 (1994).

${ }^{17}$ A. I. Larkin and Yu. N. Ovchinnikov, J. Low Temp. Phys. 21, 409 (1979).

${ }^{18}$ M. Konczykowski, S. Demirdiş, C. J. van der Beek, R. Prozorov, M. Tanatar, P. C. Canfield, S. Kasahara, T. Shibauchi, and Y. Matsuda, Bull. Am. Phys. Soc. 56, 1 (2011).

${ }^{19}$ L. Fang, Y. Jia, J. A. Schlueter, A. Kayani, Z. L. Xiao, H. Claus, U. Welp, A. E. Koshelev, G. W. Crabtree, and W. K. Kwok, Phys. Rev. B 84, 140504(R) (2011).

${ }^{20}$ S. Kasahara, T. Shibauchi, K. Hashimoto, K. Ikada, S. Tonegawa, R. Okazaki, H. Shishido, H. Ikeda, H. Takeya, K. Hirata, T. Terashima, and Y. Matsuda, Phys. Rev. B 81, 184519 (2010).

${ }^{21}$ L. A. Dorosinskii, M. V. Indenbom, V. I. Nikitenko, Yu A. Ossip'yan, A. A. Polyanskii, and V. K. Vlasko-Vlasov, Physica C 203, 149 (1992). 
${ }^{22}$ C. P. Bean, Phys. Rev. Lett. 8, 6 (1962).

${ }^{23}$ E. Zeldov, J. R. Clem, M. McElfresh, and M. Darwin, Phys. Rev. B 49, 9802 (1994).

${ }^{24}$ E. H. Brandt, Phys. Rev. B 54, 4246 (1996).

${ }^{25}$ A. Soibel, E. Zeldov, M. L. Rappaport, Y. Myasoedov, T. Tamegai, S. Ooi, M. Konczykowski, and V. Geshkenbein, Nature (London) 406, 282 (2000).

${ }^{26}$ M. Konczykowski, C. J. van der Beek, M. A. Tanatar, V. Mosser, Yoo Jang Song, Yong Seung Kwon, and R. Prozorov, Phys. Rev. B 84, 180514(R) (2011).

${ }^{27}$ Y. Fasano and M. Menghini, Supercond. Sci. Technol. 21, 023001 (2008).

${ }^{28}$ E. Zeldov, A. I. Larkin, V. B. Geshkenbein, M. Konczykowski, D. Majer, B. Khaykovich, V. M. Vinokur, and H. Shtrikman, Phys. Rev. Lett. 73, 1428 (1994).

${ }^{29}$ Ernst Helmut Brandt, Phys. Rev. B 60, 11939 (1999).

${ }^{30}$ C. P. Bean and J. D. Livingston, Phys. Rev. Lett. 12, 14 (1964).

${ }^{31}$ C. J. van der Beek, M. Konczykowski, A. Abaloshev, I. Abal'osheva, P. Gierlowski, S. J. Lewandowski, M. V. Indenbom, and S. Barbanera, Phys. Rev. B 66, 024523 (2002).

${ }^{32}$ V. B. Geshkenbein, M. V. Feigel'man, and V. M. Vinokur, Physica (Amsterdam) C 212, 2511 (1991).

${ }^{33}$ C. J. van der Beek, G. J. Nieuwenhuys, P. H. Kes, H. G. Schnack, and R. P. Griessen, Physica C 197, 320 (1992); H. G. Schnack, J. G. Lensink, R. Griessen, C. J. van der Beek, and P. H. Kes, ibid. 197, 337 (1992)

${ }^{34}$ M. P. Maley, J. O. Willis, H. Lessure, and M. E. McHenry, Phys. Rev. B 42, 2639 (1990).

${ }^{35}$ Y. Abulafia, A. Shaulov, Y. Wolfus, R. Prozorov, L. Burlachkov, Y. Yeshurun, D. Majer, E. Zeldov, and V. M. Vinokur, Phys. Rev. Lett. 75, 2404 (1995).

${ }^{36}$ M. Kończykowski, C. J. van der Beek, M. A. Tanatar, Huiqian Luo, Zhaosheng Wang, Bing Shen, Hai Hu Wen, and R. Prozorov, Phys. Rev. B 86, 024515 (2012).
${ }^{37}$ R. Prozorov, M. A. Tanatar, R. T. Gordon, C. Martin, H. Kim, V. G. Kogan, N. Ni, M. E. Tillman, S. L. Bud'ko, and P. C. Canfield, Physica (Amsterdam) C 469, 582 (2009).

${ }^{38}$ K. Hashimoto, K. Cho, T. Shibauchi, S. Kasahara, Y. Mizukami, R. Katsumata, Y. Tsuruhara, T. Terashima, H. Ikeda, M. A. Tanatar, H. Kitano, N. Salovich, R. W. Giannetta, P. Walmsley, A. Carrington, R. Prozorov, and Y. Matsuda, Science 22, 1554 (2012).

${ }^{39}$ R. Prozorov, M. A. Tanatar, Bing Shen, Peng Cheng, Hai-Hu Wen, S. L. Bud'ko, and P. C. Canfield, Phys. Rev. B 82, 180513 (2010).

${ }^{40}$ M. Grisolia, S. Demirdis, C. J. van der Beek, Y. Fasano, A. Forget, and D. Colson (unpublished).

${ }^{41}$ K. Hashimoto, M. Yamashita, S. Kasahara, Y. Senshu, N. Nakata, S. Tonegawa, K. Ikada, A. Serafin, A. Carrington, T. Terashima, H. Ikeda, T. Shibauchi, and Y. Matsuda, Phys. Rev. B 81, 220501 (2010).

${ }^{42}$ H. Shishido, A. F. Bangura, A. I. Coldea, S. Tonegawa, K. Hashimoto, S. Kasahara, P. M. C. Rourke, H. Ikeda, T. Terashima, R. Settai, Y. Onuki, D. Vignolles, C. Proust, B. Vignolle, A. McCollam, Y. Matsuda, T. Shibauchi, and A. Carrington, Phys. Rev. Lett. 104, 057008 (2010).

${ }^{43}$ A. Oral, J. C. Barnard, S. J. Bending, S. Ooi, H. Taoka, T. Tamegai, and M. Henini, Phys. Rev. B 56, R14295 (1997).

${ }^{44}$ Yanina Fasano, J. Herbsommer, and F. de la Cruz, Phys. Status Solidi B 215, 563 (1999).

${ }^{45}$ Yanina Fasano, M. Menghini, F. de la Cruz, and G. Nieva, Phys. Rev. B 62, 15183 (2000).

${ }^{46}$ A. Soibel, Y. Myasoedov, M. L. Rappaport, T. Tamegai, S. S. Banerjee, and E. Zeldov, Phys. Rev. Lett. 87, 167001 (2001).

${ }^{47}$ Ming Li, P. H. Kes, S. F. W. R. Rycroft, C. J. van der Beek, and M. Konczykowski, Physica C 408-410, 25 (2004).

${ }^{48}$ C. J. Olson, C. Reichhardt, and Franco Nori, Phys. Rev. Lett. 80, 2197 (1998).

${ }^{49}$ Ernst Helmut Brandt and Shi-Ping Zhou, Physics 2, 22 (2009); Ernst Helmut Brandt and Mukunda P. Das, arXiv:1007.1107.

${ }^{50}$ M. Rotter, C. Hieke, and D. Johrendt, Phys. Rev. B 82, 014513 (2010). 